\title{
AUTOESTIMA DE IDOSOS COMUNITÁRIOS E FATORES ASSOCIADOS: ESTUDO DE BASE POPULACIONAL*
}

Michelle Couto Salerno ${ }^{1}$, Alisson Fernandes Bolina ${ }^{2}$, Flavia Aparecida Dias ${ }^{3}$, Nayara Paula Fernandes Martins ${ }^{3}$, Darlene Mara
dos Santos Tavares ${ }^{4}$

${ }^{1}$ Discente de Enfermagem. Universidade Federal do Triângulo Mineiro. Uberaba, MG, Brasil.

${ }^{2}$ Enfermeiro. Doutorando em Enfermagem. Universidade de São Paulo. Ribeirão Preto, SP, Brasil.

${ }^{3}$ Enfermeira. Doutoranda em Atenção à Saúde. Universidade Federal do Triangulo Mineiro. Uberaba, MG, Brasil.

${ }^{4}$ Enfermeira. Doutora em Enfermagem. Docente da Universidade Federal do Triângulo Mineiro. Uberaba, MG, Brasil.

RESUMO: Objetivou-se avaliar autoestima de idosos comunitários e verificar sua associação com variáveis sociodemográficas e de saúde. O inquérito epidemiológico ocorreu na zona urbana de Uberaba, estado de Minas Gerais, no qual participaram 980 idosos. Utilizaram-se Escalas de Autoestima de Rosenberg, Katz, Lawton e Depressão Geriátrica Abreviada e análise descritiva com testes de Mann Whitney, Kruskal Wallis, correlação de Spearman e regressão linear múltipla $(p<0,05)$. A coleta de dados ocorreu entre agosto de 2012 e março de 2013. Predominou o sexo feminino, 70-80 anos, casados, renda de um salário mínimo, quatro a sete anos de estudo, que moravam com filhos. A mediana foi de cinco doenças. Os escores de autoestima apresentaram mediana de 10. Os preditores dos piores níveis de autoestima foram maior idade $(p<0,01)$, menor escolaridade $(p=0,021)$ e indicativo de depressão $(p<0,001)$. Os idosos comunitários apresentaram elevada autoestima e o indicativo de depressão foi o preditor principal da baixa autoestima.

DESCRITORES: Autoestima; Idoso; Atenção à saúde do idoso; Enfermagem geriátrica.

\section{SELF-ESTEEM OF COMMUNITY-BASED ELDERLY AND ASSOCIATED FACTORS: A POPULATION-BASED STUDY}

ABSTRACT: The aim was to assess the self-esteem of community-based elderly and verify its association with socio-demographic and health variables. The epidemiological survey was held in the urban region of Uberaba, a city in the state of Minas Gerais, with 980 elderly people. The Rosenberg Self-Esteem, Katz, Lawton and Short Geriatric Depression Scales were used and, for descriptive analysis, the Mann-Whitney and Kruskal-Wallis tests, Spearman's correlation and multiple linear regression were used $(p<0.05)$. The data were collected between August 2012 and March 2013. The female gender was predominant, 70-80 years, married, income one minimum wage, between four and seven years of education, who lived with their children. The median number of illnesses was five. The median selfesteem score was ten. The predictors of worse levels of selfesteem were higher age $(p<0.01)$, lower education $(p=0.021)$ and signs of depression $(p<0.001)$. The community-based elderly presented high self-esteem and signs of depression were the main predictor of low self-esteem.

DESCRIPTORS: Self-esteem; Aged; Elderly health care; Geriatric nursing.

\section{AUTOESTIMA DE ANCIANOS COMUNITARIOS Y FACTORES ASOCIADOS: ESTUDIO DE BASE POPULACIONAL}

RESUMEN: Estudio cuyo objetivo fue evaluar la autoestima de ancianos comunitarios y verificar su asociación con variables sociodemográficas y de salud. La investigación epidemiológica ocurrió en la zona urbana de Uberaba, estado de Minas Gerais. Participaron 980 ancianos. Fueron utilizadas Escalas de Autoestima de Rosenberg, Katz, Lawtone Depresión Geriátrica Abreviada y análisis descriptivo con testes de Mann Whitney, Kruskal Wallis, correlación de Spearman y regresión linear múltipla $(p<0,05)$. Los datos fueron obtenidos entre agosto de 2012 y marzo de 2013. Fueron predominantes el sexo femenino, 70-80 años, casados, renta de un salario mínimo, cuatro a siete años de estudio, que vivían con hijos. La mediana fue de cinco enfermedades. Los scores de autoestima presentaron mediana de 10. Los predictores de los peores niveles de autoestima fueron mayor edad $(p<0,01)$, menor escolaridad $(p=0,021)$ e indicativo de depresión $(p<0,001)$. Los ancianos comunitarios presentaron elevada autoestima y el indicativo de depresión fue el predictor principal de la baja autoestima. DESCRIPTORES: Autoestima; Anciano; Atención a la salud del anciano; Enfermería geriátrica.

*Artigo proveniente de Trabalho de Conclusão de Curso intitulado: "Autoestima de idosos e seus fatores associados." Universidade Federal do Triângulo Mineiro, 2013.

\section{Autor Correspondente:}

Darlene Mara dos Santos Tavares

Universidade Federal do Triangulo Mineiro

R. Jonas de Carvalho, 420 - 38055-440 - Uberaba, MG, Brasil

E-mail: darlenetavares@enfermagem.uftm.edu.br
Recebido: $25 / 06 / 2015$ Finalizado: 10/09/2015 


\section{INTRODUÇÃO}

A autoestima pode ser definida como "o sentimento, o apreço e a consideração que uma pessoa sente por si própria, ou seja, o quanto ela gosta de si, como ela se vê e o que pensa sobre ela mesma" $^{\prime(1: 48)}$. Esses sentimentos são construídos no decorrer da vida de acordo com as experiências adquiridas $^{(2)}$.

Na população idosa, as situações de desafetos e rejeições no âmbito familiar e social propiciam o desenvolvimento da baixa autoestima. Somado a isto, as próprias limitações físicas, psicológicas, sociais e ambientais advindas com o avançar da idade podem contribuir para esta condição(3). Entretanto, os idosos podem desenvolver estratégias para melhorar a autoestima, como por exemplo, a valorização dos aspectos positivos do envelhecimento bem como os conhecimentos adquiridos com o passar dos anos ${ }^{(4)}$.

Estudo nacional verificou elevada autoestima entre idosos frequentadores de um Centro de Atenção Integral à Saúde $(M d=36)^{(5)}$. Em outro estudo, a autoestima associou-se à percepção positiva da saúde $(p=0,003)$ e os idosos do sexo masculino com 70 anos e mais obtiveram maior média em relação aos de 60 a 69 anos $(p=0,04)$. Não se observou relação da autoestima com o estado conjugal $(p=0,590)$, escolaridade $(p=0,316)$, sexo $(p=0,352)$ e renda $(p=0,602)^{(2)}$.

Pesquisa internacional com idosos da comunidade obteve que o arranjo domiciliar se associou à autoestima; aqueles que residiam com a família possuíam níveis mais elevados de autoestima em comparação aos que moravam sozinhos $(p<0,001)^{(6)}$. Adicionalmente, a literatura científica têm sugerido que a depressão, o número de doenças e a incapacidade funcional afetam negativamente a autoestima ${ }^{(2,7)}$.

No entanto, os inquéritos brasileiros sobre a temática foram realizados com população idosa que frequentava centros de convivência ${ }^{(2,5)}$. Este fato pode contribuir para a elevada autoestima verificada nos achados, em decorrência da possibilidade de socialização. Nesta perspectiva, denota-sea necessidade doaprofundamento desta temática com idosos brasileiros da comunidade por meio de estudos epidemiológicos de base populacional.

Em sendo a autoestima um sentimento que auxilia na superação das limitações física, psicológica e social, decorrentes do avançar da idade $^{(3)}$, a identificação dos aspectos relacionados a essa condição nos idosos comunitários poderá fornecer subsídios aos profissionais de saúde da atenção primária à saúde para o planejamento do processo de promoção da saúde, tendo em vista o envelhecimento saudável.

Assim, objetivou-se avaliar os escores de autoestima de idosos comunitários e verificar a sua associação com as variáveis sociodemográficas e de saúde.

\section{MÉTODO}

Trata-se de um estudo epidemiológico de base populacional, com delineamento transversal, observacional e analítico, realizado na zona urbana de um município no interior de Minas Gerais.

O cálculo amostral populacional foi realizado considerando-se $95 \%$ de confiança, $80 \%$ de poder do teste, margem de erro de $4 \%$ para as estimativas intervalares e proporção estimada de $\varpi=0,5$ para as proporções de interesse. Partiu-se de uma amostra de 2.149 idosos.

Os critérios de inclusão foram: ter 60 anos ou mais de idade; não apresentar declínio cognitivo e residir na zona urbana do município de Uberaba, estado de Minas Gerais. Atenderam aos critérios estabelecidos 980 idosos, sendo que foram excluídos aqueles que recusaram (37) participar do estudo; encontravam-se hospitalizados (14); faleceram (266); não foram encontrados após três visitas consecutivas (183); mudaram de endereço (193); outros motivos (316). Destaca-se que 160 idosos apresentaram declínio cognitivo.

Os dados foram coletados no domicílio no período de agosto de 2012 a março de 2013. Para a caracterização dos dados socioeconômicos e demográficos e de saúde foi utilizado instrumento estruturado construído pelo Grupo de Pesquisa em Saúde Coletiva.

Para mensurar a autoestima foi utilizada a Escala de Autoestima de Rosenberg ${ }^{(1)}$, que contém 10 itens distribuídos aleatoriamente. Cada item é atribuído um valor de 0 a 3, com o resultado da soma de todas as questões variando de 0 a 30 pontos $^{(1)}$, no qual o menor escore associa-se a elevada autoestima e trinta pontos faz referência à mais baixa autoestima.

A capacidade funcional foi obtida pelas escalas deKatz (1963) eLawton (1969), validadas no Brasil( ${ }^{(8-9)}$. A Escala de Katz modificada é constituída por seis questões objetivas que investigam as Atividades 
Básicas de Vida Diária (ABVD). Para cada tarefa investigada são ofertadas alternativas, ou seja, se os idosos realizam de forma independente ou não. Cada resposta positiva equivale a um ponto, de forma que ao final os idosos são classificados em independentes para as ABVD quando atingem 5-6 pontos; dependência moderada, 3-4; e dependência importante, $0-2^{(8)}$.

A escala de Lawton é composta de nove Atividades Instrumentais da Vida Diária (AIVD). Para cada questão existem três alternativas: realiza sem ajuda (3 pontos), realiza com ajuda parcial (2 pontos), não consegue realizar (1 ponto). A pontuação máxima da escala é 27 sendo a classificação funcional:27 a19 pontos, considerado independente; de 18 a 10, dependência parcial; e abaixo de nove, apresenta-se dependente ${ }^{(9)}$.

O indicativo de depressão foi avaliado pela Escala de Depressão Geriátrica Abreviada, adaptado à realidade no Brasil ${ }^{(10)}$. Essa escala é constituída por 15 questões objetivas; considerase positivo para indicativo de depressão quando o escore for superior a cinco pontos ${ }^{(10)}$.

As variáveis estudadas foram: sexo; faixa etária, em anos (60-70, $70-80$ e 80 ou mais); estado conjugal (nunca se casou ou morou com companheiro(a); mora com esposo(a) ou companheiro(a); viúvo(a); separado(a), desquitado(a) ou divorciado(a); escolaridade, em anos de estudo (sem escolaridade, $1-4,4-8,9+11$, $\geq 11$ ); renda individual mensal, em salários mínimo (sem renda, $<1,1,1\} 3,3-5,>5$ ); arranjo de moradia (mora só, com cuidador profissional, somente com o cônjuge, com outro de sua geração, com filhos, com netos, outros arranjos), número de morbidades $(1-4,4-6, \geq 6)$, capacidade funcional nas ABVD (tomar banho, vestir-se, higiene pessoal, transferência, continência e alimentação) e AIVD (telefone, fazer compras, cuidar das finanças, preparar as próprias refeições, arrumar a casa, fazer trabalhos manuais domésticos, lavar e passar roupa, tomar medicação corretamente e sair para lugares distantes usando algum meio de transporte), número de morbidades $(1+4$, $4 \nmid 6, \geq 6$ ), autopercepção de saúde (péssima, má, regular, boa, ótima) e indicativo de depressão (sim, não).

Foi construída uma planilha eletrônica no programa Excel $^{\oplus}$. Os dados coletados foram processados em duas bases de dados, que foram submetidas à consistência. Quando se verificou dados inconsistentes, retornou-se ao formulário de coleta original para correção. Posteriormente, os dados foram transportados para o software
Statiscal Package for Social Sciences (SPSS) versão 17.0 para proceder as análises.

Realizou-se análise descritiva por meio de distribuição de frequências absolutas e percentuais. Para a verificação dos fatores associados à autoestima, utilizou-se análise bivariada preliminar. As variáveis nominais foram recategorizadas tornando-as dicotômicas: estado conjugal (sem ou com companheiro), renda (sem ou com), arranjo domiciliar (mora só ou não), indicativo de depressão (sim e não) e incapacidade funcional para ABVD (sim ou não) e AIVD (sim e não). A autopercepção de saúde também foi recategorizada (péssima-má, regular, boa-ótima). A normalidade dos dados foi verificada pelo teste de Kolmogorov Smirnov. Os testes utilizados foram Mann Whitney, Kruskal Wallis e correlação de Spearman. As variáveis que apresentaram $\mathrm{p}<0,1$ foram inseridas no modelo multivariado por regressão linear múltipla. Neste modelo foram consideradas significativas quando $\mathrm{p}<0,05$.

Estudo aprovado pelo Comitê de Ética em Pesquisa com Seres Humanos da Universidade Federal do Triângulo Mineiro, sob protocolo $\mathrm{n}^{\circ}$ 2.265. Os idosos assinaram o Termo de Consentimento Livre e Esclarecido antes da coleta dos seus dados.

\section{RESULTADOS}

O maior percentual dos idosos $(\mathrm{n}=980)$ era do sexo feminino $(64,3 \%)$; com $70-80$ anos $(50 \%)$; casados $(42,6 \%)$ seguidos pelos viúvos $(42 \%) ; 4$-8 anos de estudo (34,5\%), renda individual mensal de um salário mínimo (50,2\%) e morava com filhos $(35,1 \%)$.

A Tabela 1, a seguir, apresenta as características sociodemográficas e econômicas da população estudada.

Prevaleceram os idosos independentes em todas as ABVDs $(95,8 \%)$, sendo que as atividades com maior percentual de dependentes foram continência $(3 \%)$ e banho $(1,5 \%)$.

Nas AIVDs prevaleceram aqueles com dependência parcial $(69,6 \%)$; com maior percentual de idosos dependentes nas atividades: viagens $(28 \%)$ e trabalho doméstico $(19,1 \%)$.

O maior percentual dos idosos apresentou boa autopercepção de saúde $(40,8 \%)$ seguida de regular $(40,2 \%)$. O indicativo de depressão representou 25,6\%. Na Tabela 2, a seguir, 
Tabela 1 - Distribuição de frequência das variáveis sociodemográficas e econômicas dos idosos. Uberaba, MG, Brasil, 2013

\begin{tabular}{|c|c|c|}
\hline Variáveis & $\mathbf{N}$ & $\%$ \\
\hline \multicolumn{3}{|l|}{ Sexo } \\
\hline Feminino & 630 & 64,3 \\
\hline Masculino & 350 & 35,7 \\
\hline \multicolumn{3}{|l|}{ Faixa etária (em anos) } \\
\hline 60 - 70 & 283 & 28,9 \\
\hline $70+80$ & 490 & 50 \\
\hline 80 anos ou mais & 207 & 21,1 \\
\hline \multicolumn{3}{|l|}{ Estado conjugal } \\
\hline $\begin{array}{l}\text { Nunca se casou ou morou com } \\
\text { companheiro }\end{array}$ & 48 & 4,9 \\
\hline Mora com companheiro & 417 & 42,6 \\
\hline Viúvo & 412 & 42 \\
\hline Separado/desquitado/divorciado & 103 & 10,5 \\
\hline \multicolumn{3}{|l|}{ Escolaridade (em anos) } \\
\hline Sem escolaridade & 212 & 21,6 \\
\hline 1 1-4 & 274 & 28 \\
\hline 4 -8 & 338 & 34,5 \\
\hline 8 & 48 & 4,9 \\
\hline 9卜-11 & 25 & 2,6 \\
\hline 11 ou mais & 83 & 8,5 \\
\hline \multicolumn{3}{|l|}{ Renda individual (em salários mínimo) } \\
\hline Sem renda & 82 & 8,4 \\
\hline$<1$ & 15 & 1,5 \\
\hline 1 & 492 & 50,2 \\
\hline $1-3$ & 310 & 31,6 \\
\hline $3-5$ & 48 & 4,9 \\
\hline$>5$ & 30 & 3,1 \\
\hline Não responderam & 3 & 0,3 \\
\hline \multicolumn{3}{|l|}{ Arranjo de Moradia } \\
\hline Só & 181 & 18,5 \\
\hline Somente com cuidador profissional & 4 & 0,4 \\
\hline Somente com o cônjuge & 221 & 22,6 \\
\hline $\begin{array}{l}\text { Com outros de sua geração (com ou } \\
\text { sem cônjuge) }\end{array}$ & 83 & 8,5 \\
\hline Com filhos (com ou sem cônjuge) & 344 & 35,1 \\
\hline Com netos (com ou sem cônjuge) & 76 & 7,8 \\
\hline Outros arranjos & 70 & 7,1 \\
\hline Não responderam & 1 & 0,1 \\
\hline
\end{tabular}

apresentam-se as características de saúde da população estudada.

Os idosos apresentaram mediana de cinco doenças associadas, variando de zero a 17. A mediana dos escores de autoestima foi dez, considerada elevada, variando de zero a 24.
Tabela 2 - Distribuição de frequência das variáveis de saúde dos idosos. Uberaba, MG, Brasil, 2013

\begin{tabular}{lcc} 
Variáveis & $\mathbf{n}$ & $\%$ \\
\hline ABVDs & & \\
\hline $\begin{array}{l}\text { Independência em todas as seis } \\
\text { funções }\end{array}$ & 939 & 95,8 \\
$\begin{array}{l}\text { Dependente em pelo menos uma } \\
\text { função }\end{array}$ & 41 & 4,2 \\
\hline AIVDs & 6 & 0,6 \\
\hline Dependência total & 682 & 69,6 \\
\hline Dependência parcial & 292 & 29,8 \\
\hline Independente & & \\
\hline Autopercepção de saúde* & 28 & 2,9 \\
\hline Péssima & 56 & 5,7 \\
\hline Má & 394 & 40,2 \\
\hline Regular & 400 & 40,8 \\
\hline Boa & 101 & 10,3 \\
\hline Ótima & & \\
\hline Indicativo de depressão & 251 & 25,6 \\
\hline Sim & 729 & 74,4
\end{tabular}

Para verificar os fatores associados à autoestima, realizou-se, inicialmente, análise bivariada. As variáveis que atenderam ao critério estabelecido $(p<0,1)$ foram inseridas no modelo de regressão linear múltipla: sexo $(Z=-2,357 ; p=0,0180)$, idade $(r=0,113 ; \quad p<0,001)$, estado conjugal $(\beta=-2,526$; $p=0,012)$, escolaridade $(r=-0,114 ; p<0,001)$, renda $(\beta=-2,344 ; p=0,019)$, capacidade funcional para ABVD $(Z=-4,938 ; \quad p<0,001)$ e AIVD $(\beta=-2,769$; $\mathrm{p}=0,006)$, autopercepção de saúde $(\mathrm{H}=-87,409$; $\mathrm{p}<0,001)$, indicativo de depressão $(Z=-16,364$; $\mathrm{p}<0,001)$ e número de morbidades $(\mathrm{r}=-0,194$; $p<0,001)$.

A Tabela 3, a seguir, apresenta a análise multivariada de regressão linear dos fatores associados à autoestima entre os idosos do município de Uberaba, 2013.

$\mathrm{Na}$ análise multivariada, obteve-se como preditores dos piores níveis de autoestima: maior idade $(\beta=0,07 ; p<0,01)$, menor escolaridade $(\beta=-0,065 ; p=0,021)$, presença de indicativo de depressão $(\beta=-0,407 ; p<0,001)$ e autopercepção de saúde péssima-má ( $\beta=0110 ; p<0,001)$ e regular ( $\beta=0120 ; p<0,001)$. O indicativo de depressão foi o maior preditor da pior autoestima. 
Tabela 3 - Modelo de Regressão dos fatores associado à autoestima de idosos. Uberaba, MG, Brasil, 2013

\begin{tabular}{|c|c|c|c|c|}
\hline \multirow[b]{2}{*}{ Variáveis } & \multicolumn{2}{|c|}{ Modelo Inicial } & \multicolumn{2}{|c|}{ Modelo Final } \\
\hline & $\beta$ & $p$ & $\beta$ & $p$ \\
\hline Sexo feminino & 0,035 & 0,254 & - & - \\
\hline Idade & 0,067 & 0,024 & 0,07 & 0,01 \\
\hline $\begin{array}{l}\text { Ausência de } \\
\text { companheiro }\end{array}$ & 0,005 & 0,871 & - & - \\
\hline $\begin{array}{l}\text { Ausência de } \\
\text { escolaridade }\end{array}$ & $-0,063$ & 0,027 & $-0,065$ & 0,021 \\
\hline $\begin{array}{l}\text { Ausência de } \\
\text { renda }\end{array}$ & 0,011 & 0,685 & - & - \\
\hline $\begin{array}{l}\text { Incapacidade } \\
\text { funcional para } \\
\text { ABVD }\end{array}$ & 0,055 & 0,056 & - & - \\
\hline $\begin{array}{l}\text { Incapacidade } \\
\text { funcional para } \\
\text { AIVD }\end{array}$ & 0,006 & 0,643 & - & - \\
\hline $\begin{array}{l}\text { Presença de } \\
\text { indicativo de } \\
\text { depressão }\end{array}$ & $-0,395$ & $<0,001$ & $-0,407$ & $<0,001$ \\
\hline $\begin{array}{l}\text { Autopercepção } \\
\text { da saúde } \\
\text { (péssima/má) }\end{array}$ & 0,092 & 0,004 & 0,11 & $<0,001$ \\
\hline $\begin{array}{l}\text { Autopercepção } \\
\text { da saúde } \\
\text { (regular) }\end{array}$ & 0,117 & $<0,001$ & 0,121 & $<0,001$ \\
\hline $\begin{array}{l}\text { Número de } \\
\text { doenças }\end{array}$ & 0,022 & 0,482 & - & - \\
\hline
\end{tabular}

\section{DISCUSSÃO}

A maioria de mulheres neste estudo reflete os dados nacionais $(55,7 \%)$, podendo estar relacionado às maiores taxas de mortalidade entre os homens ${ }^{(11)}$. Por outro lado os resultados evidenciados em relação à faixa etária divergem dos achados do Brasil em que, do total de idosos, $55,5 \%$ tinham $60-70$ anos $^{(11)}$. Esta divergência pode estar relacionada ao fato de que população do presente inquérito vem sendo acompanhada pelo Grupo de Pesquisa em Saúde Coletiva desde 2005.

O predomínio de idosos casados foi semelhante a inquérito conduzido em São Paulo-SP no qual $58,9 \%$ dos idosos moravam com companheiro ${ }^{(12)}$. O percentual de viúvos também corrobora com investigação com idosos usuários da rede pública de saúde em Natal-RN $(40 \%)^{(3)}$.

No país, o arranjo domiciliar predominante tem sido de idosos morando com os filhos $(30,7 \%)$ (11), resultado semelhante a esta investigação.
As estimativas nacionais também foram corroborantes no que se refere ao rendimento individual mensal, em que a maioria dos idosos $(38,6 \%)$ recebiam entre meio e um salário mínimo ${ }^{(11)}$; salienta-se que à época da coleta dos dados, o valor correspondente ao salário era de $\mathrm{R} \$ 678,00^{(13)}$.

Concernente às características de saúde da população estudada, o maior percentual de dependentes nas ABVDs vai ao encontro de inquérito realizado com idosos de Lafaiete Coutinho-BA em que prevaleceram: controle de esfíncter $(8,5 \%)$, principalmente incontinência urinária $(7,6 \%)$, e tomar banho (5,4\%). Entretanto, nas AIVDs as porcentagens foram superiores para a dependência quando comparadas a essa mesma investigação (41\%). Os autores identificaram que as atividades com maior proporção de dependentes foram o uso do telefone $(36,6 \%)$ e ir a lugares sozinho $(25,1 \%)^{(14)}$, divergindo do presente estudo.

O percentual de idosos com boa autopercepção de saúde foi inferior aos inquéritos em CampinasSP $(64,2 \%)^{(12)}$. Este fato pode ser sido influenciado pelo percentual de idosos desta investigação que apresentam comprometimento funcional e morbidades associadas.

A prevalência de indicativo de depressão corrobora com o obtido em Montes Claros-MG $(20,9 \%)^{(15)}$. Acredita-se que este resultado tenha relação com o número de doenças associadas entre os idosos.

Os escores de autoestima levantados nesta investigação $(M D=10,0)$ foram convergentes ao encontrado em inquérito conduzido em São Paulo-SP com um grupo de idosos frequentadores de um Ambulatório Fisioterapêutico $(M D=9,91)$ (16) e de usuários da rede pública de saúde em Natal-RN $(M D=9,37)^{(3)}$. Destaca-se que a literatura científica mostra que a autoestima é um dos principais preditores de envelhecimento bemsucedido entre os idosos. A prática da enfermagem sugere que as ações realizadas sejam capazes de promover uma melhora da autoestima visando maior grau de adaptabilidade ao envelhecimento, apesar de problemas de saúde e questões pessoais, nesta fase da vida ${ }^{(17)}$.

A despeito dos fatores associados aos piores níveis de autoestima, a maior idade associouse à baixa autoestima divergindo de estudo realizado em Florianópolis-SC $(p=0,05)^{(4)}$. Sabese que algumas alterações podem surgir com o envelhecimento, afetando a autoestima. Dentre 
estas, destaca-se a dificuldade da memória que vem sendo relatada por idosos como um dos fatores que interfere negativamente nessa condição(4).

Assim, os profissionais de saúde devem desenvolver atividades educativas em grupo visando criar espaços de discussão e reflexão sobre os aspectos relacionados à senescência e senilidade. Tais atividades, além de favorecer a socialização, podem contribuir no empoderamento desses indivíduos frente aos desafios impostos do próprio processo de envelhecimento, minimizando o seu impacto na autoestima.

Em contrapartida, nesta mesma investigação, os autores sugerem que o acréscimo da autoestima de acordo com o aumento da faixa etária possivelmente esteja relacionado à aceitação das mudanças inerentes ao próprio processo de envelhecimento ${ }^{(4)}$. Somado a isso, com o decorrer da idade, os idosos podem desenvolver mecanismos de adaptação, através da valorização dos aspectos positivos dessa fase da vida e dos conhecimentos adquiridos com o passar dos $\operatorname{anos}^{(4)}$.

A menor escolaridade foi um preditor de baixa autoestima, mas o mesmo não se observou em inquérito conduzido em Santa Catarina com idosos ativos fisicamente $(p=0,316)^{(2)}$. No entanto, destaca-se que a aprendizagem contribui para o desenvolvimento do indivíduo e instiga na busca por novos conhecimentos, principalmente daqueles que não tiveram a oportunidade de estudar. Essa busca de conhecimentos afeta positivamente a autoestima de idosos ${ }^{(4)}$. O nível de escolaridade associa-se ao acesso à informação, a melhores oportunidades sociais ao longo da vida, bem como facilita a oferta de serviços de saúde e consequentemente melhores condições de vida ${ }^{(5)}$.

A relação de indicativo de depressão e a autoestima corrobora com resultados demonstrados em pesquisa entre idosos praticantes de atividade física em FlorianópolisSC, que observou que quanto mais elevados os sintomas depressivos, menor a autoestima $(p<0,001)^{(1)}$. Este fato também tem sido evidenciado em situações de diagnóstico clínico da doença, como demonstrado em inquérito conduzido em Santa Catarina, em que idosos com depressão obtiveram menor média de autoestima comparados aos que não apresentavam a sintomatologia clínica da doença $(p=0,04)^{(18)}$.
É mister que o profissional de saúde durante a assistência aos idosos busque atentar para o reconhecimento do indicativo de depressão. A detecção precoce e o tratamento adequado podem amenizar o impacto dessa morbidade tanto nos aspectos relacionados à saúde quanto na autoestima. Para isso, ressalta-se a escala de depressão geriátrica como um instrumento de rastreio válido e de fácil utilidade na prática clínica ${ }^{(10)}$. Devem-se encaminhar os indivíduos com avaliação positiva para confirmação diagnóstica e acompanhamento profissional especializado.

A baixa autoestima relacionada à pior percepção de saúde corrobora com investigação desenvolvida em Santa Catarina, em que idosos com avaliação positiva de sua saúde obtiveram melhores níveis de autoestima comparados àqueles com autopercepção negativa $(p=0,003)^{(2)}$. Tal resultado pode estar relacionado à existência de problemas de saúde entre aqueles com percepção negativa de saúde, que repercute em limitações nas atividades diárias ${ }^{(2)}$, interferindo negativamente na autoestima.

Sabe-se que o aspecto físico apresenta uma estreita relação com o estado emocional e psicológico, entretanto, indivíduos com doenças crônicas e que estão sensibilizados para o controle de sua condição de saúde conseguem desenvolver equilíbrio que satisfaça o caminho do envelhecimento saudável ${ }^{(19)}$. O enfermeiro, portanto, pode desenvolver estratégias de promoção da saúde com essa população visando ao empoderamento para o autocuidado e monitoramento do estado de saúde. Nesse sentido, a equipe de saúde deve atentar para as necessidades psicobiológicas, condições de saúde e de vida do idoso visando a contribuir no seu cuidado ${ }^{(20)}$.

\section{CONCLUSÃO}

O levantamento apontou que os idosos comunitários apresentaram elevada autoestima e as variáveis que estiveram associadas à baixa autoestima foram: maior idade, menor escolaridade, presença de indicativo de depressão e autopercepção de saúde péssima e regular. Vale ressaltar que o indicativo de depressão foi o preditor principal dessa condição.

Acredita-se que este estudo pode contribuir para ampliar o conhecimento de fatores associados à autoestima de idosos comunitários, entretanto, não se pode concluir efeito de 
causalidade entre as variáveis investigadas devido ao desenho transversal. Outra limitação é a escassez de pesquisas científicas sobre a temática, especialmente na população idosa geral, dificultando a discussão dos resultados deste estudo. Sugerem-se outros estudos de delineamento longitudinal, preferencialmente em escala ampliada, visando verificar os fatores que impactam na autoestima de idosos da comunidade.

\section{APOIO FINANCEIRO}

Fundação de Amparo à Pesquisa do Estado de Minas Gerais (FAPEMIG) e Conselho Nacional de Desenvolvimento Científico e Tecnológico (CNPq).

\section{REFERÊNCIAS}

1. Dini GM, Quaresma MR, Ferreira LM. Adaptação cultural e validação da versão brasileira da escala de auto-estima de Rosenberg. Rev Soc Bras Cir Plástica. [Internet] 2004; 19(1) [acesso em 09 jun 2014]. Disponível: http://www.rbcp.org.br/imageBank/ PDF/19-01-04pt.pdf

2. Antunes G, Mazo GZ, Balbe GP. Relação da autoestima entre a percepção de saúde e aspectos sociodemográficos de idosos praticantes de exercício físico. Rev Educ Fis UEM [Internet] 2011; 22(4) [acesso em 11 jun 2014]. Disponível: https://dx.doi.org/10.4025/ reveducfis.v22i 4.10825

3. Ferreira CL, Santos LMO, Maia EMC. Resiliência em idosos atendidos na Rede de Atenção Básica de Saúde em município do nordeste brasileiro. Rev Esc Enferm USP [Internet] 2012; 46(2) [acesso em 10 jun 2014]. Disponível: http://dx.doi.org/10.1590/S008062342012000200009

4. Meurer ST, Benedetti TRB, Mazo GZ. Aspectos da autoimagem e autoestima de idosos ativos. Motriz ver. educ. fís. [Internet] 2009; 15(4) [acesso em 10 jun 2014]. Disponível: http://cev.org.br/biblioteca/aspectosautoimagem-autoestima-idosos-ativos/

5. Costa ECS, Andrade GMM, Jinkings YF, Corrêa RGCF, Aquino DMC. Avaliação da auto-estima em idosos de um Centro Integral à Saúde do Idoso - São LuísMA. Rev Pesq Saude [Internet] 2011; 12(1) [acesso em 09 jun 2014]. Disponível: http://www.huufma.br/site/ estaticas/revista_hu/pdf/Revista_jan-abril-2011.pdf

6. Sok SR, Yun EK. A comparison of physical health status, self-esteem, family support and healthpromoting behaviours between aged living alone and living with family in Korea. J Clin Nurs [Internet] 2011; 20(11-12) [acesso em 10 jun 2014]. Disponível: http:// dx.doi.org/10.1111/j.1365-2702.2010.03551.x
7. Meurer ST, Borges LJ, Benedetti TRB, Mazo GZ. Associação entre sintomas depressivos, motivação e autoestima de idosos praticantes de exercícios físicos. Rev Bras Cienc Esporte [Internet] 2012; 34(3) [acesso em 11 jun 2014]. Disponível: http://dx.doi.org/10.1590/ S0101-32892012000300011

8. Lino VTS, Pereira SRM, Camacho LAB, Ribeiro Filho ST, Buksman S. Adaptação transcultural da Escala de Independência em Atividades da Vida Diária (Escala de Katz). Cad Saude Publica. [Internet] 2008; 24(1) [acesso em 11 jun 2014]. Disponível: http://dx.doi.org/10.1590/ S0102-311X2008000100010

9. Santos RL, Virtuoso Júnior JS. Confiabilidade da versão brasileira da Escala das Atividades Instrumentais da Vida Diária. Rev Bras P Saúde [Internet]. 2008; 21(4) [acesso em 10 jun 2014]. Disponível: http://ojs.unifor. br/index.php/RBPS/article/download/575/2239

10. Almeida OP, Almeida SA. Confiabilidade da versão brasileira da Escala de Depressão em Geriatria (GDS) versão reduzida. Arq Neuropsiquiatr [Internet]. 1999; 57(n.2B) [acesso em 10 jun 2014]. Disponível: http:// dx.doi.org/10.1590/S0004-282X1999000300013

11. Instituto Brasileiro de Geografia e Estatística (IBGE). Síntese de Indicadores Sociais: uma análise das condições de vida da população brasileira. Brasília-DF: Ministério do Planejamento, Orçamento e Gestão [Internet]. 2012 [acesso em 18 mar 2014]. Disponível: http://www.ibge.gov.br/home/estatistica/ populacao/condicaodevida/indicadoresminimos/ sinteseindicsociais2012/default_tab_pdf.shtm

12. Lima MG, Barros MBA, César CLG, Goldbaum M, Carandina L, Ciconelli RM. Impact of chronic disease on quality of life among the elderly in the state of São Paulo, Brazil: a population-based study. Rev Panam Salud Publica [Internet]. 2009; 25(4) [acesso em 20 jun 2014]. Disponível: http://dx.doi.org/10.1590/S102049892009000400005

13. Departamento intersindical de estatística e estudos socioeconômicos (Dieese). Cesta básica nacional salário mínimo nominal e necessário. [Internet]. 2013 [acesso em 10 jun 2014]. Disponível: http://www. dieese.org.br/analisecestabasica/salarioMinimo.html

14. Freitas RS, Fernandes $M H$, Coqueiro RS, Reis Júnior WM, Rocha SV, Brito TA. Capacidade funcional e fatores associados em idosos: estudo populacional. Acta Paul Enferm [Internet]. 2012; 25(6) [acesso em 20 jun 2014]. Disponível: http://dx.doi.org/10.1590/S010321002012000600017

15. Hoffmann EJ, Ribeiro F, Farnese JM, Lima EWB. Sintomas depressivos e fatores associados entre idosos residentes em uma comunidade no norte de Minas Gerais, Brasil. J Bras Psiquiatr [Internet]. 2010; 59(3) [acesso em 20 jun 2014]. Disponível: http://dx.doi. org/10.1590/S0047-20852010000300004 
16. Chaim J, Izzo H, Sera CTN. Cuidar em saúde: satisfação com imagem corporal e auto-estima de idosos. O Mundo da Saúde [Internet]. 2009; 33(2) [acesso em 08 jun 2014]. Disponível: http://www. saocamilo-sp.br/pdf/mundo_saude/67/175a181.pdf

17. Cha NH, Seo EJ, Sok SR. Factors influencing the successful aging of older Korean adults. Contemp Nurse. 2012; 41(1):78-87.

18. Mazo GZ, Krug RR, Virtuoso JF, Streit IA, Benetti MZ. Autoestima e depressão em idosos praticantes de exercícios físicos. Revista Kinesis. [Internet]. 2012; 30(1) [acesso em 20 ago 2014]. Disponível: http://cascavel. ufsm.br/revistas/ojs-2.2.2/index.php/kinesis/article/ download/5724/3399

19. Silva ACS, Santos I, Berardinelli LMM. Body image of the elderly in the reflex of self-care for healthy aging: A social-poetic study. Online Braz J of Nursing [Internet]. 2010; 9(1) [acesso em 09 jun 2014]. Disponível: http:// dx.doi.org/10.5935/1676-4285.20102779

20. Garbaccio JL, Garcia TF, Cândida DA. Avaliação da independência de idosos atendidos por uma estratégia de saúde da família. Cogitare enferm. [Internet]. 2013; 18(4) [acesso em 11 jun 2015]. Disponível: http://dx.doi. org/10.5380/ce.v18i4.34914 